\title{
Ultrafast Gain Dynamics in Quantum Dot Amplifiers: Theoretical Analysis and Experimental Investigations
}

Poel, Mike van der; Gehrig, Edeltraud; Hess, Ortwin; Birkedal, Dan; Hvam, Jørn Märcher

Published in:

I E E E Journal of Quantum Electronics

Link to article, DOI:

10.1109/JQE.2005.852795

Publication date:

2005

Document Version

Publisher's PDF, also known as Version of record

Link back to DTU Orbit

Citation (APA):

Poel, M. V. D., Gehrig, E., Hess, O., Birkedal, D., \& Hvam, J. M. (2005). Ultrafast Gain Dynamics in Quantum Dot Amplifiers: Theoretical Analysis and Experimental Investigations. I E E E Journal of Quantum Electronics, 41(9), 1115-1123. https://doi.org/10.1109/JQE.2005.852795

\section{General rights}

Copyright and moral rights for the publications made accessible in the public portal are retained by the authors and/or other copyright owners and it is a condition of accessing publications that users recognise and abide by the legal requirements associated with these rights.

- Users may download and print one copy of any publication from the public portal for the purpose of private study or research.

- You may not further distribute the material or use it for any profit-making activity or commercial gain

- You may freely distribute the URL identifying the publication in the public portal 


\title{
Ultrafast Gain Dynamics in Quantum-Dot Amplifiers: Theoretical Analysis and Experimental Investigations
}

\author{
Mike van der Poel, Edeltraud Gehrig, Ortwin Hess, Dan Birkedal, and Jørn M. Hvam
}

\begin{abstract}
Ultrafast gain dynamics in an optical amplifier with an active layer of self-organized quantum dots (QDs) emitting near $1.3 \mu \mathrm{m}$ is characterized experimentally in a pump-probe experiment and modeled theoretically on the basis of QD Maxwell-Bloch equations. Experiment and theory are in good agreement and show ultrafast subpicoseconds gain recovery followed by a slower 5 ps recovery. This behavior is found to be mainly caused by longitudinal optical phonon scattering and strongly dependents on electronic structure and confinement energy of the dots. A low amplitude-phase coupling ( $\alpha$ factor) is theoretically predicted and demonstrated in the experiments. The fundamental analysis reveals the underlying physical processes and indicates limitations to QD-based devices.
\end{abstract}

Index Terms-Quantum dots (QDs), semiconductor optical amplifiers (SOAs), ultrafast optics.

\section{INTRODUCTION}

$\mathbf{Q}$ UANTUM-DOT (QD)-based optoelectronic devices have been intensively studied in recent years. The reason for the interest in this type of device lies in the numerous highly desirable and promising properties that result from the specific physical properties of the dots, e.g., their discrete energy levels. In particular, these are high differential gain and low laser threshold current [1], low alpha factor [2], and ultrafast modulation properties [3]. Today, real-world QD-based optoelectronic devices have shown very low threshold current densities [4], low alpha factors [5], high gain over a large bandwidth [6], and 10-GHz direct modulation of a QD-based laser [7]-[8]. All in all, the field is reaching an advanced state.

This paper presents the first comparison of measured ultrafast gain/absorption dynamics with detailed theoretical analysis of calculations based on spatially resolved QD Maxwell-Bloch equations that take into account the waveguiding structure, the spatial dependence and microscopic coupling between light and carriers as well as relaxation via carrier-carrier and carrier-phonon scattering. Furthermore this comparison is carried out on self-assembled InAs-InGaAs-GaAs QDs emitting near

Manuscript received February 8, 2005; revised May 13, 2005. This work was supported in part by the EU-IST project DOTCOM (Project no. IST-200028713).

M. van der Poel and J. M. Hvam are with the Research Center COM, NanoDTU, Technical University of Denmark, Lyngby DK-2800, Denmark (e-mail: mvp@com.dtu.dk; hvam@com.dtu.dk).

E. Gehrig and O. Hess are with the Advanced Technology Institute School of Electronics and Physical Sciences, University of Surrey, Surrey GU2 7XH, U.K. (e-mail: e.gehrig@ surrey.ac.uk; o.hess@ surrey.ac.uk).

D. Birkedal is with the Research Center Communication, Optics and Materials (COM), NanoDTU, Technical University of Denmark, Lyngby DK-2800, Denmark and also with the Alight Technologies, Farum DK-3520, Denmark (e-mail: db@alight.dk).

Digital Object Identifier 10.1109/JQE.2005.852795 the important $1310-\mathrm{nm}$ telecommunications wavelength. The experimental studies have been performed with a pump-probe setup with heterodyne detection.

\section{EXPERIMENT AND SAMPLE DETAILS}

Experimental characterization of ultrafast properties of a QD SOA was carried out using a degenerate pump-probe experiment with heterodyne detection [9]. The laser source consists of a Ti:sapphire oscillator, regenerative amplifier, and optical parametric amplifier in cascade and provides transform limited pulses with 150-ps full-width half-maximum (FWHM) duration and tuneable wavelength in the 950-2000 nm range. The heterodyne detection allows simultaneous measurement of the probe's amplitude and phase which in turn reflect the SOAs gain and index of refraction. These two quantities yield complementary information: the gain is a measurement of the population inversion at the probed energy while the index is related to the off-resonant gain profile through the Kramers-Kronig relations.

The sample under investigation is a ridge-waveguide semiconductor optical amplifier (SOA) with an active layer of five sheets of self-organized InAs dots overgrown with $5 \mathrm{~nm}$ $\mathrm{In}_{0.15} \mathrm{Ga}_{0.85} \mathrm{As}$ [10]. Each dot layer is separated by a GaAs spacer layer of $30 \mathrm{~nm}$ thickness and has a QD density of $4 \cdot 10^{10} \mathrm{~cm}^{-2}$. Ridge width is $7 \mu \mathrm{m}$ and length is $2 \mathrm{~mm}$. The facets are uncoated but have a 7 degree angle to reduce feedback and hence inhibit lasing. Insertion loss of this device was measured to be $18 \mathrm{~dB}$ and this loss is mainly due to in- and outcoupling losses. The reason for this high coupling loss is due to a highly asymmetric mode profile in the far field. Amplified spontaneous emission (ASE) spectra [see Fig. 1(a)] from these deeply confining dots show a ground-state emission centered on $1260 \mathrm{~nm}$ with an inhomogeneous broadening of $96 \mathrm{meV}$ and an excited-state emission centered on $1190 \mathrm{~nm}$ appearing at high injection current. The ground state confinement energy is $290 \mathrm{meV}$. The SOA gain for different input pulse energies and bias currents is shown in Fig. 2 for a pulse wavelength of $1235 \mathrm{~nm}$. The pulse energies are measured before incoupling to the waveguide. In the linear regime we see a gain ranging from about $-13 \mathrm{~dB}$ at zero bias to $4 \mathrm{~dB}$ at injection of $25 \mathrm{~mA}$ current. As pulse energy increases, we see a saturation of the amplifier leading to bleaching of the absorption and decrease of the gain. At even higher input energies two-photon absorption (TPA) drives the gain downward for all bias currents. It is seen from Fig. 2 that transparency at this wavelength is between 15 and $20 \mathrm{~mA}$. The dots have a pyramidal shape, with typical base length of $14 \mathrm{~nm}$ [10]. Details on the electronic structure of this kind of QD have been calculated by Stier et al. [11] and are 


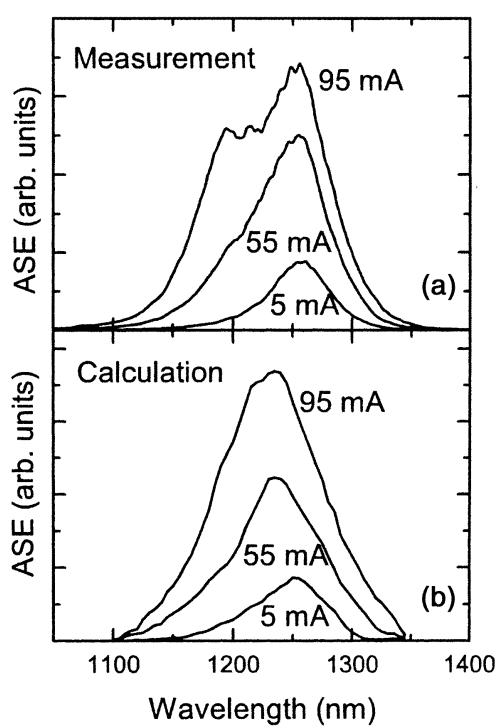

Fig. 1. (a) Measured and (b) calculated ASE spectra for the QD SOA at different injenction current levels.

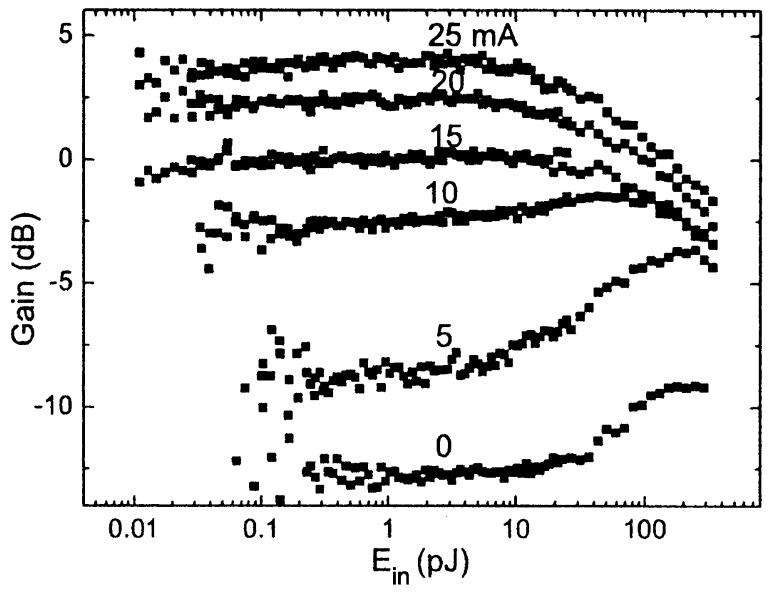

Fig. 2. Saturation of pulse gain for the QD SOA at $1235 \mathrm{~nm}$.

used as input for the modeling described below. In the given example, the dots contain three electron and five hole levels.

\section{THEORETICAL DESCRIPTION}

In the following, we summarize the mesoscopic theoretical description [12] that is used for the numerical modeling of the ultrafast gain and index dynamics in QD lasers. The spatially resolved QD Maxwell-Bloch equations mesoscopically describe the spatiotemporal light field and inter/intra-level carrier dynamics in each dot of a typical QD ensemble in QD lasers. In particular, this includes the influence of material properties (dot size, level energies, dot distribution), spontaneous luminescence, counterpropagation of amplified spontaneous emission and induced recombination as well as carrier diffusion in the wetting layer of the QD laser. Intradot scattering via emission and absorption of phonons, as well as the scattering with the carriers and phonons of the surrounding wetting layer are dynamically included on a mesoscopic level [12]. Spatial fluctuations in size and energy levels of the QDs and irregularities in the spatial distribution of the QDs in the active layer are simulated

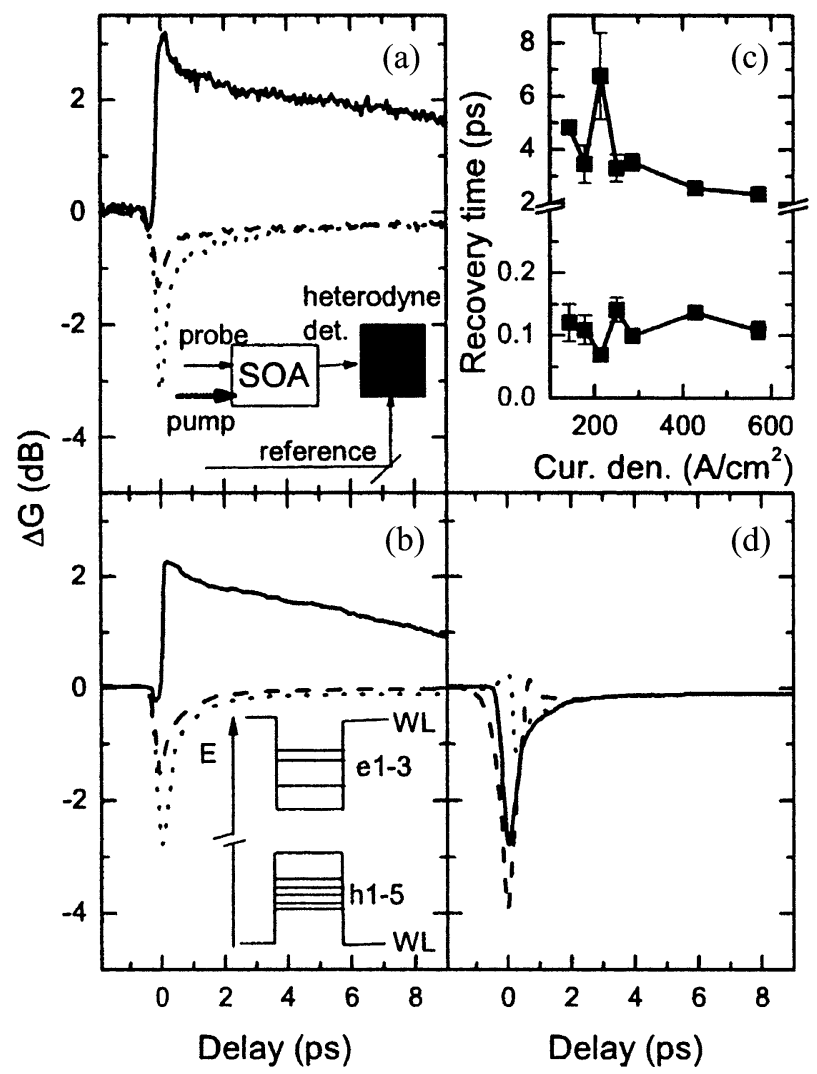

Fig. 3. (a) Measured and (b) calculated gain change during amplification of a short pulse at $1273 \mathrm{~nm}$ for an injected current density 0 (solid line), $86 \mathrm{~A} / \mathrm{cm}^{2}$ (dashed line), and $571 \mathrm{~A} / \mathrm{cm}^{2}$ (dotted line). (c) $1 / e$ recovery times from bi-exponential fit to the gain recovery as function of current density. (d) Calculated gain change at $1273 \mathrm{~nm}$ and current density of $571 \mathrm{~A} / \mathrm{cm}^{2}$ with both phonon and Auger contributions (solid line), phonon-only contribution (dashed), and Auger-only contribution (dotted). The modeled QD energy level scheme is shown schematically as an inset in (b). The electron and hole states are labeled e1-3 and h1-5, respectively, from the most confined state to the least confined state.

via statistical methods. The spatial fluctuations are modeled in the form $X_{\mathrm{QD}}=X_{\mathrm{QD}}^{\mathrm{av}}\left(1+X_{\mathrm{QD}}^{\mathrm{fluc}}\right)$ where $X_{\mathrm{QD}}^{\mathrm{av}}$ denotes the average value and $X_{\mathrm{QD}}^{\mathrm{fluc}}$ is the spatially dependent fluctuation that is simulated with a Gaussian distribution. This leads to spatially dependent energy corrections and damping rates. The material properties of the QD ensemble matrix are considered via effective parameters [11]. The energies of the electrons are 180,100, and $85 \mathrm{meV}$; the energies of the holes are 110, 90, 75, 70, and $55 \mathrm{meV}$ relative to the conduction band and valence band edges in the wetting layer, respectively. The inset of Fig. 3(b) shows these levels schematically. The time-dependent calculation of the carrier and light field dynamics allow for explicit consideration of the individual time scales of the various interaction processes. The relevant time scales range from the femtosecond regime (for the fast carrier scattering processes) up to the picosecond and nanosecond regime (for the dynamics of the propagating light fields and of the spatial carrier density).

The QD Maxwell Bloch equations consist of QD semiconductor Bloch equations (for the carrier dynamics in each QD), a diffusion equation for the carriers in the embedding material and a wave equation for the counterpropagating light fields. The QD semiconductor Bloch equations describe the dynamic changes of the electron (e, level index $i^{e}$ ) and hole (h, level index $i^{h}$ ) 
distributions in each energy level of the dot and the dynamics of the interlevel dipoles $p$ (for each combination of electron and hole energy levels)

$$
\begin{aligned}
\frac{\partial n^{\mathrm{e}, \mathrm{h}}\left(i^{\mathrm{e}, \mathrm{h}}\right)}{\partial t}= & \Lambda^{\mathrm{e}, \mathrm{h}}\left(i^{\mathrm{e}, \mathrm{h}}\right)+g^{\mathrm{e}, \mathrm{h}}\left(i^{e, h}\right)-\gamma_{\mathrm{nr}} n^{\mathrm{e}, \mathrm{h}}\left(i^{\mathrm{e}, \mathrm{h}}\right) \\
& -\sum_{i^{\mathrm{h}, \mathrm{e}}} \Gamma_{\mathrm{sp}} n^{\mathrm{e}, \mathrm{h}}\left(i^{\mathrm{e}, \mathrm{h}}\right) \cdot n^{\mathrm{h}, \mathrm{e}}\left(i^{\mathrm{h}, \mathrm{e}}\right) \\
& +\left.\frac{\partial n^{\mathrm{e}, \mathrm{h}}\left(i^{\mathrm{e}, \mathrm{h}}\right)}{\partial t}\right|_{\mathrm{QD}} ^{\mathrm{e}, \mathrm{h}-\mathrm{ph}}+\left.\frac{\partial n^{\mathrm{e}, \mathrm{h}}\left(i^{\mathrm{e}, \mathrm{h}}\right)}{\partial t}\right|_{\mathrm{QD}-\mathrm{WL}} \\
\frac{\partial p^{ \pm}\left(i^{\mathrm{e}}, i^{\mathrm{h}}\right)}{\partial t}= & -\left(\mathrm{i} \bar{\omega}\left(i^{\mathrm{i}}, i^{\mathrm{h}}\right)+\gamma_{\mathrm{p}}\right) p^{ \pm}\left(i^{\mathrm{e}}, i^{\mathrm{h}}\right) \\
& -\frac{\mathrm{i}}{\hbar}\left[n^{\mathrm{e}}\left(i^{\mathrm{e}}\right)+n^{\mathrm{h}}\left(i^{\mathrm{h}}\right)\right] \mathcal{U}^{ \pm} \\
& -\frac{\mathrm{i}}{\hbar} \delta \mathcal{U}_{\mathrm{nl}}^{ \pm}+F_{\mathrm{p}}+\left.\frac{\partial p^{ \pm}\left(i^{\mathrm{i}}, i^{\mathrm{h}}\right)}{\partial t}\right|_{\mathrm{QD}} ^{\mathrm{p}-\mathrm{ph}}
\end{aligned}
$$

The pump term

$$
\begin{aligned}
& \left.\Lambda^{\mathrm{e}, \mathrm{h}}\left(i^{e, h}\right)\right)=\Gamma_{\mathrm{QDS}}(I \eta / e d)\left(n_{\mathrm{eq}}^{\mathrm{e}, \mathrm{h}}\left(i^{e, h}\right) /\right. \\
& \qquad \sum_{l} n_{\mathrm{eq}}^{\mathrm{e}, \mathrm{h}}\left(i^{e, h}\left(D^{\mathrm{e}, \mathrm{h}}\left(i^{e, h}\right)-n^{\mathrm{e}, \mathrm{h}}\left(i^{e, h}\right)\right)\right)
\end{aligned}
$$

mesoscopically represents the carrier injection and includes the pump-blocking effect. It depends on the absolute injection current $I$, pump efficiency $\eta$, and the thickness of the active area $d$. $D^{\mathrm{e}, \mathrm{h}}\left(i^{e, h}\right)$ denotes the degeneracy of an end energy level (i.e., the maximum occupation with carriers). $\Gamma_{\mathrm{QDS}}$ describes the reduction of the pump efficiency resulting from the vertically arranged QDs, i.e., the "spatial overlap" between carrier injection and a vertical stack of QDs in the medium. $g^{\mathrm{e}, \mathrm{h}}$ is the generation rate for induced recombination, $\Gamma_{\mathrm{sp}}$ includes the spontaneous carrier recombination, and $\gamma_{\mathrm{nr}}$ is the rate due to nonradiative recombination. $\partial n^{\mathrm{e}, \mathrm{h}} /\left.\partial t\right|_{\mathrm{QD}} ^{\mathrm{c}-\mathrm{ph}}$ and $\partial n^{\mathrm{e}, \mathrm{h}} /\left.\partial t\right|_{\mathrm{QD}-\mathrm{WL}}$ describe carrier-carrier and carrier-phonon scattering for the intradot relaxation and the interaction with the wetting layer, respectively. The scattering rates for carrier-phonon intradot relaxation generally include emission ("-") and absorption ("+") of longitudinal acoustical (LA) phonons, longitudinal optical (LO) phonons and multiphonon processes $( \pm 2 \mathrm{LO}, \pm 2 \mathrm{LA}$, and $\pm \mathrm{LO} \pm \mathrm{LA})$. They can be derived on the basis of quantum kinetic equations of motion for the single particle density matrices with respect to the carrier phonon Hamiltonian [12]. The matrix elements for the respective carrier-phonon interactions as well as the scattering rates for Auger processes are calculated according to the derivations in [13]-[15]. Elastic Coulomb scattering processes between the QDs and the wetting layer leading to dynamic shifts in line shapes an emission frequencies are treated on the basis of perturbation theory [16], [17]. The generation rates given by

$$
\begin{aligned}
& g^{\mathrm{e}, \mathrm{h}}\left(i^{\mathrm{e}, \mathrm{h}}\right)=\operatorname{Re}\left[(\mathrm{i} / \hbar) \sum_{i^{\mathrm{h}, \mathrm{e}}}\right. {\left[\left(\mathcal{U}^{+} p^{+*}\left(i^{\mathrm{e}}, i^{\mathrm{h}}\right)+\mathcal{U}^{-} p^{-*}\left(i^{\mathrm{e}}, i^{\mathrm{h}}\right)\right)\right.} \\
&\left.\left.-\left(\mathcal{U}^{+*} p^{+}\left(i^{\mathrm{e}}, i^{\mathrm{h}}\right)+\mathcal{U}^{-*} p^{-}\left(i^{\mathrm{e}}, i^{\mathrm{h}}\right)\right)\right]\right]
\end{aligned}
$$

depend on the interlevel polarization $p$ and on the optical field contributions of spontaneous and induced emission constituting the local field $\mathcal{U}^{ \pm}$. The Langevin noise term $F_{\mathrm{p}}$ describes dipole fluctuations [12]. The local fields $\mathcal{U}^{ \pm}=\vec{d}\left(i^{\mathrm{e}}, i^{\mathrm{h}}\right) \vec{E}^{ \pm}+\delta \mathcal{U}^{ \pm}$ are composed of the optical light field contributions $E^{ \pm}$as well as those induced by Coulomb screening in each QD and by the Coulomb interactions between the carriers in the QD and the carriers in the wetting layer, $\delta \mathcal{U} . d\left(i^{\mathrm{e}}, i^{\mathrm{h}}\right)$ is the interlevel dipole matrix element [11] and $\tau_{\mathrm{p}}$ denotes the dephasing time of the interlevel dipole. The interlevel polarization depends via $\bar{\omega}\left(i^{\mathrm{e}}, i^{\mathrm{h}}\right)=\hbar^{-1}\left(\mathcal{E}^{\mathrm{e}}+\mathcal{E}^{\mathrm{h}}\right)-\omega(\omega$ is the frequency of the propagating light fields) on the carrier energies.

The dynamics of the carriers in the wetting layer can be described by the following diffusion equation

$$
\begin{aligned}
\frac{\partial N_{\mathrm{WL}}^{\mathrm{e}, \mathrm{h}}}{\partial t}=\nabla D_{f}\left(\nabla N_{\mathrm{WL}}^{\mathrm{e}, \mathrm{h}}\right) & +\frac{J}{e d}+\left.\frac{\partial N^{\mathrm{e}, \mathrm{h}}}{\partial t}\right|_{\mathrm{QD}-\mathrm{WL}} \\
& -\gamma_{\mathrm{sp}} N_{\mathrm{WL}}^{\mathrm{e}, \mathrm{h}} N_{\mathrm{WL}}^{\mathrm{h}, \mathrm{e}}-\gamma_{\mathrm{WL}}^{\mathrm{nr}} N_{\mathrm{WL}}^{\mathrm{e}, \mathrm{h}}
\end{aligned}
$$

with a pump term describing carrier injection and a rate for nonradiative emission processes.

The QD semiconductor Bloch equations including the dynamic intradot scattering and the interactions with the wetting layer constitute a fundamental basis for a microscopic analysis and interpretation of the relevant physical processes such as dynamic carrier excitation and relaxation. At each location in the active dot medium the carrier dynamics is linked (via the generation rate and the dipole dynamics) to the light field dynamics. The dynamics of the counterpropagating $(+,-)$ light fields is described by [12]

$$
\begin{aligned}
\nabla_{\mathrm{T}}^{2} \vec{E}^{ \pm} \pm 2 \mathrm{i} \beta \frac{\partial}{\partial z} \vec{E}^{ \pm}+ & \frac{\partial^{2}}{\partial z^{2}} \vec{E}^{ \pm}+\frac{2 \mathrm{i} \omega}{c^{2}} \frac{\partial}{\partial t} \vec{E}^{ \pm}-\frac{1}{c^{2}} \frac{\partial^{2}}{\partial t^{2}} \vec{E}^{ \pm} \\
& =-\mu_{0} \omega^{2} \vec{P}^{ \pm}-2 \mathrm{i} \omega \frac{\partial}{\partial t} \vec{P}^{ \pm}+F_{\mathrm{E}}
\end{aligned}
$$

The Langevin noise term $F_{\mathrm{E}}$ considers spontaneous light field fluctuations. The polarization of the active semiconductor medium $P^{ \pm}=V^{-1} \sum_{i^{\mathrm{e}}, i^{\mathrm{h}}} d\left(i^{\mathrm{e}}, i^{\mathrm{h}}\right) p^{ \pm}\left(i^{\mathrm{e}}, i^{\mathrm{h}}\right)$ is the source of the optical fields ( $V$ denotes the normalization volume of the crystal).

In the following, we will present numerical and experimental results on the propagation of an ultrashort (150 fs) pulse in a QD amplifier. We will focus on ultrafast dynamics (Section IV), influence of dot properties (Section V) as well as on the amplitude phase coupling (Section VI). In the theory, the optical injection of a light pulse is realized via the boundary conditions of the light fields at the facets.

\section{ULTRAFAST DyNAMics}

On a microscopic level, QDs show very complex carrier dynamics. Depending on the material system and the physical properties of the individual dots, a hierarchy of carrier scattering processes via emission and absorption of phonons, multiphonon processes as well as Auger processes may occur. In our pump-probe experiment the pump-induced change in the SOAs gain and index is measured at the exit facet at the pump-probe energy. This measurement can be simulated by appropriate spatial and energetical integration.

\section{A. From Absorption to Gain}

Fig. 3(a) shows experimental results on the pump-probe gain dynamics at the QD ground state emission energy for different 
injected current densities. The pump energy of $20 \mathrm{pJ}$ is close to the saturation input energy (see Fig. 2) while the two orders of magnitude weaker probe is in the linear regime. For our analysis of the gain dynamics, we have subtracted the TPA contribution (occurring when pump and probe arrive simultaneously) from the pump-probe signal. The TPA contribution is estimated by measuring gain compression at transparency current since no net stimulated emission or absorption occur in that case. Depending on carrier injection, the pump pulse is absorbed and probe pulse transmission is increased due to absorption bleaching (corresponding to positive values in Fig. 3) or the pump pulse is amplified and the probe pulse transmission is reduced due to gain compression (corresponding to negative values in Fig. 3) thereby dynamically 'shaping' the available gain via the nonlinear light-matter coupling. The theoretical gain recovery curves [Fig. 3(b)] are derived from the (spatially averaged) microscopic distribution of the dipole density that is dynamically calculated within the framework of the QD Bloch equations. The level scheme of the three electron and five hole states in the modeled QDs is indicated in Fig. 3(b). The imaginary part of the interlevel polarization is directly correlated to the carrier-induced spatiospectral gain the signal pulse experiences during its propagation. The results of the computational modeling and the measurement are in good agreement and reveal the underlying physical processes which will be detailed in the following.

At zero bias current, the absorption bleaching that is observed, is characterized by an initial subps decay of small amplitude followed by a slower decay with a decay time of 40 ps. The fast component originates from the ultrafast relaxation of the initial nonthermal carrier distribution toward a quasi-equilibrium state defined by the energetic level separation of the ground and excited levels and the corresponding phononassisted level-dynamics. The second, longer time is a combination of carrier lifetime and carrier escape from the dot. The amplification regime is characterized by a strong carrier depletion at the probed energy levels. This "level hole burning" leads to a large pump-induced gain compression. In order to analyze the individual influence of phonon and Auger processes, we have performed additional simulations where we reduced the scattering terms in the QD Bloch equations to either phonon scattering or Auger processes only [Fig. 3(d)]. The results clearly demonstrated that for the given QD system the specific shape of the gain dynamics is mostly determined by phonon interactions. The Auger contribution is comparatively small, e.g., one third of the phonon contribution (depending on injection current density). However, Auger processes do play a role and additionally modify the gain shape. The intradot scattering (primarily via emission and absorption of phonons) of the gain typically occurs on timescales of a few hundred femtoseconds up to a few picoseconds leading to a fast partial recovery of the gain. The temporal regime after approximately 1 ps is characterized by a comparatively slow re-establishment of the inversion via carrier capture due to phonon scattering. A phononmediated carrier capture time on the order of $4 \mathrm{ps}$ is consistent with the calculations of Magnusdottir et al. [18] and is further supported experimentally by the capture time's weak dependence of injected carrier density in the gain regime as shown in Fig. 3(c).
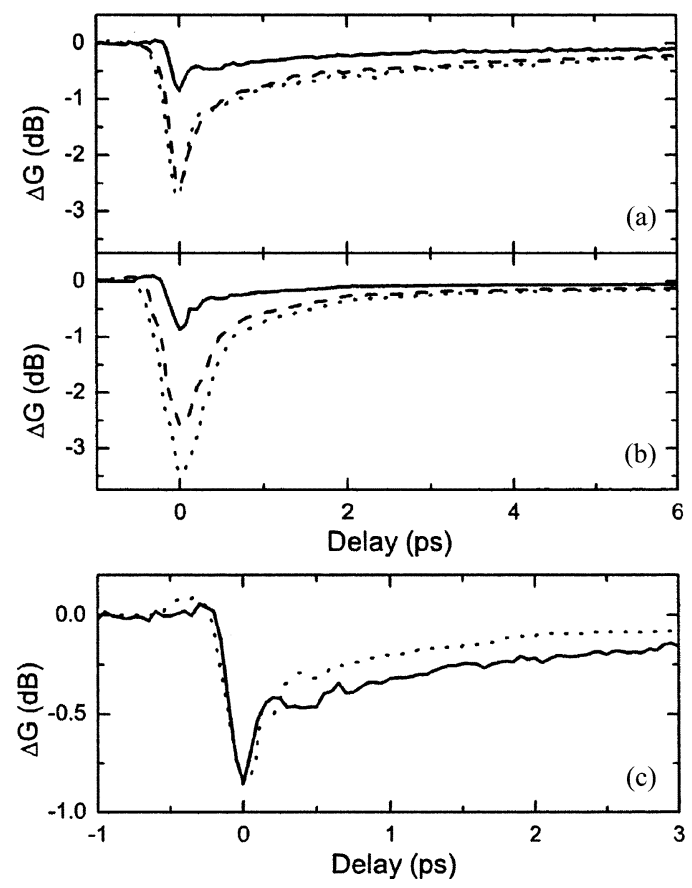

Fig. 4. (a) Measured and (b) calculated gain change during amplification of a short pulse at $1216 \mathrm{~nm}$ (solid line), $1250 \mathrm{~nm}$ (dashed line), and $1270 \mathrm{~nm}$ (dotted line) for an injected current density of $570 \mathrm{~A} / \mathrm{cm} 2$. (c) Measured (solid line) and calculated (dotted line) gain changes near transparency $(1216 \mathrm{~nm})$ shown on an enlarged scale.

\section{B. Spectral Dependence of Gain Dynamics}

Fig. 4(a) shows experimental results on the dynamics of the gain for three different pump-probe wavelengths and at a constant injected current density of $570 \mathrm{~A} / \mathrm{cm}^{2}$. The pulse wavelength determines the "spectral overlap" and, consequently, the coupling to the level energies of the QD device. For this constant carrier injection level, the QD-device is in the amplification regime at $1270 \mathrm{~nm}$ (corresponding to 8.1 times transparency current) and approaches transparency when the pulse wavelength is reduced (in the example: 3.0 times transparency at $1216 \mathrm{~nm}$ ). The time-resolved measurement and calculation of the pulse-induced gain dynamics allow the fundamental analysis of the selective depletion and refilling of QD energy levels. It thus directly reveals the influence of material properties such as the level energies of the dots and the coupling to the embedding material on the optical response of the QD laser. Near transparency, the partial exchange of inversion via absorption and emission of phonons leads to characteristic modulations in the gain dynamics that are directly correlated to the corresponding increased oscillations in level populations within the charge carrier ensemble of the QDs [see Fig. 4(c)]. The modulation depth and period thereby depends on the energy separation of the levels as well as on injection current and input power.

The specific shape of the pulse-induced gain dynamics, as well as the various time scales of the gain recovery, are the fingerprints of a complex carrier-dynamics in the charge carrier ensemble in the dots. To elucidate this point we present numerical results on the level dynamics which-via the dependence of the intradot polarization on the level occupations-represent 


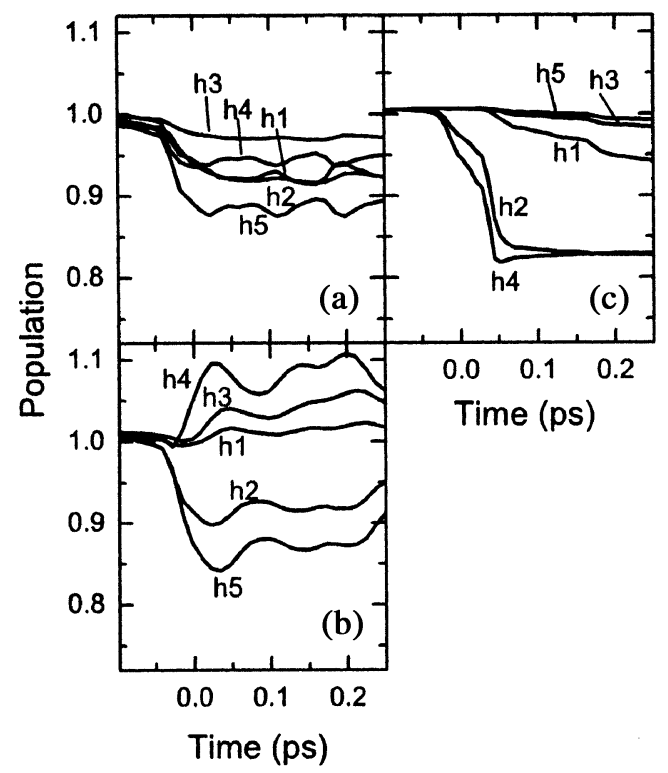

Fig. 5. Relative occupation of hole levels during the propagation of a light pulse. (a) Amplification regime. (b) Near transparency regime. (c) Amplification regime of deeply confined dot. The hole level labels indicated in the figure (h1-5) are defined in Fig. 3.

the physical origin for the gain dynamics as seen in an experiment. In the QD Maxwell-Bloch equations the inter- and intralevel dynamics are automatically represented and calculated for each (electron and hole) energy level. The calculation of the carrier distributions allows visualization of the microscopic interactions occurring within the dots. As an example, Fig. 5 visualizes the dynamics of the hole level occupation in the QDs (spatially averaged at the SOAs output facet and normalized to the occupation before the passage of the pulse) during the passage of the light pulse. The respective electron level occupations show a qualitatively similar behavior. Fig. 5(a) corresponds to the amplification regime (wavelength of the injected light pulse: $1270 \mathrm{~nm}$ ) and Fig. 5(b) to the near-transparency regime (wavelength of the injected light pulse: $1216 \mathrm{~nm}$ ), respectively. The pump pulse leads to an optical excitation of the carrier system and to a selective level-hole burning [e.g., Fig. 5(a)]. Depending on the dipole matrix elements for the individual states and depending on the frequency detuning of the pulse with respect to the frequency of the respective electron and hole transition a reduction of the individual level occupation and a partial refilling via carrier injection and microscopic scattering processes occurs. The microscopic scattering processes involved in this "level hole burning" are determined by emission and absorption of phonons, multiphonon interactions and the interaction with the carriers and phonons of the wetting layer. The magnitude of the various "channels" for relaxation mechanisms thereby depend on the QD energy levels, on the energy difference to the surrounding layers and on the coupling of a dot to its next neighbors. The dot-to-medium and dot-to-dot interactions thereby are determined by the dot density and the light fields that mesoscopically couple the QDs during their propagation. In particular, the dynamic occupation of a dot level is via carrier and phonon scattering influenced by the neighboring levels. A level energy spacing close to the LO phonon energy leads to a resonance-like exchange of occupation between neighboring levels.

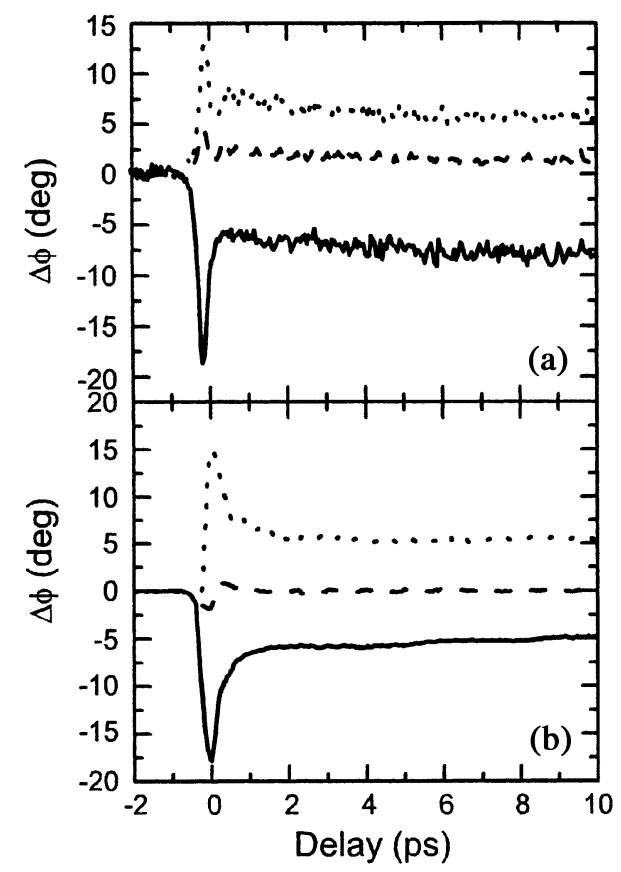

Fig. 6. (a) Measured and (b) calculated phase change during amplification of a short pulse at $1273 \mathrm{~nm}$ for an injected current density of 0 (solid line), $86 \mathrm{~A} / \mathrm{cm}^{2}$ (dashed line), and $571 \mathrm{~A} / \mathrm{cm}^{2}$ (dotted line).

Due to the dependence of the interlevel polarization on the carrier dynamics this dynamic carrier exchange directly affects the gain dynamics. This dynamic carrier exchange and level dynamics then leads to characteristic modulations that are particularly visible near transparency [Fig. 4(c)]. This behavior is most pronounced when the energy difference between the dot levels is near the LO phonon energy as is the case for our dots. More deeply confined dots with level separations much larger than this value would not display this behavior but show decoupled level dynamics and selective level hole burning instead as it can be seen in Fig. 5(c). As a consequence, the degree of coupling between the individual levels also affects the saturation behavior of a QD laser waveguide that has been optically injected with a light pulse. For the same characteristics of an injected light pulse (i.e., input power, duration, pulse shape), a QD-medium with decoupled level energies (i.e., energy separation larger than the LO phonon energy) will show a stronger saturation behavior than a QD ensemble with a strong coupling of the level energies where a mutual exchange of level occupations via scattering and relaxation dynamics occurs.

\section{Phase Dynamics}

The pulse-induced changes in the carrier distributions are-via the Bloch equations-directly reflected to changes in the polarization [see (1)]. Gain and phase changes, in turn, can be derived from the dynamics of the interlevel polarization. The measurement and calculation of the phase change thus provides complementary information to the gain dynamics. The heterodyne detection scheme in the pump-probe setup allows for the simultaneous measurement of the pump-induced change in the gain and phase of the probe pulse. Fig. 6(a) shows [for the same situation as in Fig. 3(a)] the measured phase dynamics. The results of the numerical simulation are displayed in Fig. 6(b). 
Depending on injection current density, the pulse induces a characteristic rise or decrease in the phase followed by an ultrafast partial recovery on a time scale of less than one picosecond. In the gain regime where the QD sample is inverted, the level hole-burning leads to a reduction of the carrier distributions and consequently to a rise in the induced refractive index. Immediately after the pulse the partial refilling of the dot levels (via carrier-phonon interactions) and the coupling to carriers of the wetting layer states leads to a decrease in the refractive index. In combination with the gain dynamics (compare Fig. 3) this leads to a pulse-induced maximum in the phase dynamics. According to the relation $\delta \omega=-\partial \Phi / \partial t$ the resulting decrease and increase in the instantaneous frequency within the pulse envelope can be interpreted as dynamic deceleration and acceleration of the propagating light fields. Near transparency, the dynamically varying pulse amplitude causes both induced recombination processes and carrier accumulation due to absorption. The resulting phase dynamics may thus show both, positive and negative values. For low injection current densities (i.e., absorption regime), the pulse is absorbed thereby exciting the charge carrier plasma in the dots. As a consequence, the pulse-induced phase is negative. In all current regimes, the phase dynamics immediately after the ultrafast recovery is determined by the long time behavior of the carrier distributions, gain and induced index, i.e., the (comparatively slow) filling of the dots via the injection current.

\section{InfluenCE of Material PROPERTIES: DeEP AND SHALLOW DOTS}

Next to the energy structure of the individual dot it is, in particular the energetic coupling to the environment that affects the ultrashort time dynamics of a QD ensemble. As an example we will in the following consider the dynamics of the pulse-induced gain for deep and shallow dots.

Fig. 7 visualizes the gain dynamics for deep (grey) and shallow (black) QDs in the gain regime (current density: $600 \mathrm{~A} / \mathrm{cm}^{2}$ ). Typical values for the energy structure of deep and shallow dots (leading to energy confinements of approximately 300 and $100 \mathrm{meV}$, respectively) have been taken from literature [19], [20]. The shallow dots are characterized by a small energy difference between dot states and states of the embedding material. Capture via Auger processes are in general found to be decreasing with increasing dot confinement and capture via phonon scattering is a rapidly changing function of dot size [18]. As a consequence, the gain shows a quick relaxation that is primarily determined by the ultrafast intradot carrier relaxation. The ultrafast spectroscopy on shallow dots reported by Borri et al. [19] and Akiyama et al. [21] did show a faster overall gain recovery in qualitative agreement with our calculation. In the QD ensemble consisting of deep dots the larger energy difference between dot levels and environment leads to a higher complexity in the involved scattering processes (carrier-carrier, carrier-phonon and multiphonon interactions). Thus, the gain dynamics is characterized by both the ultrafast intradot scattering that determines the shape and duration of the initial peak and a subsequent slow gain recovery due to carrier injection and the interaction between dots and wetting layers.

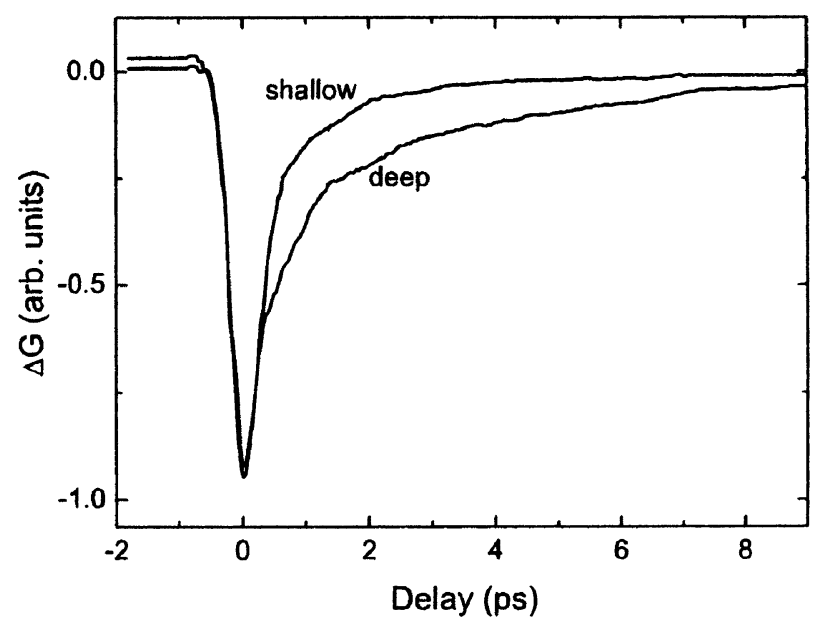

Fig. 7. Calculated gain change during the passage of a GS resonant light pulse for deep and shallow dots.

\section{INFLUENCE OF CARRIER INJECTION ON AMPlitude Phase COUPLing}

Due to their discrete level energies, QDs have a rather symmetric gain and induced index dispersion. In principle, they should thus have a small coupling of change in gain $g$ and refractive index $n$ when the carrier density $N$ is varied [22]. This coupling is usually quantified in the alpha factor, $\alpha$, which is equivalently defined in terms of the optical susceptibility $\chi$ or $g$ and $n$

$$
\alpha=\frac{\Re\left(\frac{\partial \chi}{\partial N}\right)}{\Im\left(\frac{\partial \chi}{\partial N}\right)}=-2 k \frac{\frac{\partial n}{\partial N}}{\frac{\partial g}{\partial N}}
$$

where $k$ is the light's wave number. Indeed, values near one could be found for InGaAs-GaAs QD lasers in experiments [5]. Furthermore, it could be demonstrated that the alpha factor in QD lasers is much lower and shows less spectral dispersion than in quantum-well lasers of identical material and geometry [23]. Despite the unique electronic properties of individual dots, a variety of different alpha factors arise in real ensembles of QDs. This originates from the pulse-induced nonequilibrium dynamics and the complex interplay of carrier excitation and relaxation, involving various carrier-phonon and multiphonon processes with their individual time scales. Furthermore, the spatially inhomogeneous dot distribution that typically exists in real QD devices leads to a spatial variation in shape and amplitude of the gain, index and phase dynamics. As a consequence, the measured value of the (spatially and temporally averaged) $\alpha$ may be large even though the "local" value $\alpha$ may be very small for a given time step. This effect is particularly pronounced in QD devices with a large width of the active waveguide. As an example, Fig. 8(a) shows the calculated $\alpha$ for a QD waveguide with a cavity width of $50 \mu \mathrm{m}$ at the central ground state (GS) emission wavelength as function of current injection for an ideal dot system with negligible fluctuations in dot electronic properties and a real dot device with typical dot-to-dot variations (4\% fluctuations in dot parameters). In the structure with identical dots, the strong Coulomb interactions induce a shift in the characteristic distributions of gain and index [24]. As a consequence, 


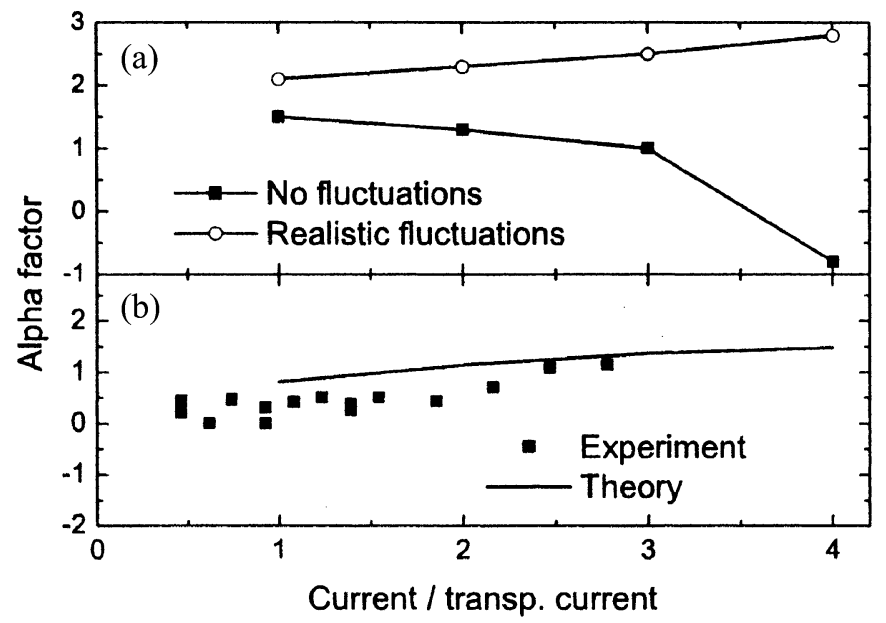

Fig. 8. (a) Theoretical alpha factor at the GS emission wavelength of $1260 \mathrm{~nm}$ for a ridge width of $50 \mu \mathrm{m}$ for two different distributions of QD properties (see text for explanation). (b) Comparison of experimental and theoretical alpha factor of $7 \mu \mathrm{m}$ ridge width SOA at GS emission wavelength.

the alpha factor decreases with current and may even become negative at high currents. In a spatially inhomogeneous dot ensemble the Coulomb-induced shift in gain and index is masked by the large inhomogeneous broadening which results in a rise in alpha with carrier density.

The alpha factor of the actual SOA (ridge width: $7 \mu \mathrm{m}$ ) was measured at the GS emission wavelength of $1260 \mathrm{~nm}$ as a function of injected current. The measurement was performed by applying small, rapid variations in the injected current while monitoring the heterodyne signal. The corresponding gain and index changes in the sample then lead to the generation of alpha-dependent sidebands in the heterodyne signal. The magnitude of alpha was extracted from an analysis of the relative height of these sidebands [25]. Thermal effects in the waveguide were eliminated by performing the current variations at a $1-\mathrm{MHz}$ speed. The measured alpha values and the results of a corresponding numerical simulation of the QD device are summarized in Fig. 8(b). Theory and experiment show a good agreement. The large inhomogeneous linewidth of the sample results in an increase in alpha with increasing current. We would like to note that the rise in alpha is small, starting from almost zero to approximately 1.3 at three times transparency current. Due to the small ridge width the influence of the lateral degree of freedom is much smaller than in a SOA with larger ridge width. As a consequence, the influence of fluctuations is decreased and the dependence on injection current is comparatively small.

A dynamical alpha factor can be inferred from the measured and calculated gain and phase dynamics (Figs. 3 and 6) and is shown in Fig. 9 for the case of zero bias current. The alpha factor dynamics is related to the redistribution of the resonantly injected carriers. In both the experiment and calculation a rapid increase in alpha is seen in the first $20 \mathrm{ps}$. After this time the dynamical alpha parameter is constant or only slowly increasing. The slow increase in the measured dynamical alpha factor after 20 ps reflects slightly different recovery times of the gain and phase. This is not reproduced in the theory which has the same

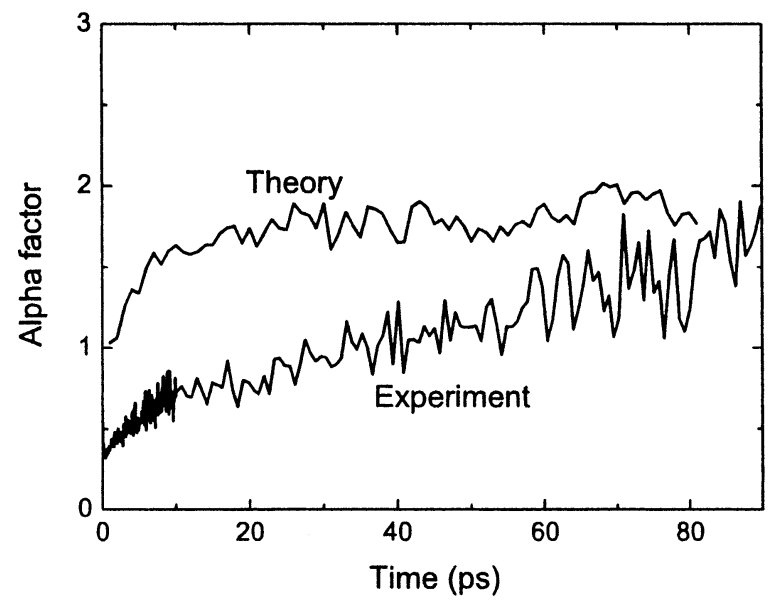

Fig. 9. Dynamical alpha factor at zero current and at GS central emission wavelength of $1260 \mathrm{~nm}$.

ultimate gain and phase recovery time. The reason for this discrepancy is not well understood. When measuring the alpha factor using optical carrier modulation rather than electrical carrier modulation a higher value is found [25], [26]. This difference is related to the very different circumstances under which the carrier density is modulated.

\section{CONCLUSION}

We have presented joint experimental and numerical results on the ultrafast gain and index dynamics in QD amplifiers. Our simulations are based on spatiotemporally resolved QD Maxwell-Bloch equations that take into account light diffraction, intradot carrier scattering as well as the dynamic interaction between dot carriers and carriers in the embedding material. The strong spatial and temporal variance in the dynamic nonlinear light-matter coupling and saturation effects occuring during the propagation of a light pulse requires the inclusion of the full spatial ad temporal dependence of the complex light field and carrier dynamics. Comparison of experimental pump-probe traces and the model shows that for typical geometries and spatially inhomogeneous dot properties the QD Maxwell-Bloch equations do give a realistic simulation of the various carrier scattering processes such as emission and absorption of phonons that are responsible for the complex gain dynamics. At small injection currents, the large level separation prevents the injected carriers from diffusing and consequently a long relaxation time of $40 \mathrm{ps}$, related to carrier lifetime, is dominating the absorption recovery. In the gain regime, however, ultrafast, subps phonon scattering is responsible for a dominating contribution to the gain recovery. The remaining gain recovery, happening on a few picoseconds timescale, is related to carrier capture from the wetting layer. The phase recovery at all currents shows a much slower recovery determined by the restoration of carrier density via the injection current. Gain dynamics is strongly dependent on dot energy level structure and comparison of the experimental data on deep dots presented in this paper with data on shallow dots presented in literature [19], [21] shows a faster gain recovery for shallow dots. This behavior is reproduced by our calculations. 
The spatiotemporally resolved calculations predict an alphaparameter decreasing with carrier density for a dot ensemble without inhomogeneous broadening. On the other hand, an inhomogeneous dot GS broadening as in the real sample results in an alpha parameter increasing with carrier density. This prediction is confirmed by experiment. The alpha value is small, increasing from ca. 0.3 to 1.3 when injection current increases from 0 to 3 times transparency current.

Our experimental and numerical results clearly demonstrate that QD lasers are characterized by ultrafast carrier processes that may be promising for high-speed dynamics. However, the spatial variance in dot properties as well as coupling between the dots and their environment will set an upper limit to e.g., signal to noise ratio in high-speed signal processing.

\section{ACKNOWLEDGMENT}

The authors would like to thank A. Kovsh of the Ioffe Institute (now at NL semiconductor) for growing the QD sample. They would also like to thank M. Laemmlin and D. Bimberg of Technische Universitt Berlin for wafer processing.

\section{REFERENCES}

[1] M. Asada, Y. Miyamoto, and Y. Suematsu, "Gain and the threshold of three-dimensional quantum-box lasers," IEEE J. Quantum Electron., vol. QE-22, no. 9, pp. 1915-1921, Sep. 1986.

[2] M. Willatzen, T. Tanaka, Y. Arakawa, and J. Singh, "Polarization dependence of optoelectronic properties in quantum dots and quantum wires-consequences of valence-band mixing," IEEE J. Quantum Electron., vol. 30, no. 3, pp. 640-653, Mar. 1994.

[3] X. Huang, A. Stintz, H. Li, L. F. Lester, J. Cheng, and K. J. Malloy, "Passive mode-locking in $1.3 \mu \mathrm{m}$ two-section InAs quantum dot lasers," Appl. Phys. Lett., vol. 78, pp. 2825-2827, 2001.

[4] G. Park, O. B. Shchekin, D. L. Huffaker, and D. G. Deppe, "Low-threshold oxide-confined 1.3-?m quantum-dot laser," IEEE Photon. Technol. Lett., vol. 13, no. 3, pp. 230-232, Mar. 2000.

[5] S. Ghosh, S. Pradhan, and P. Bhattacharya, "Dynamic characteristics of high-speed $\mathrm{In}_{0.4} \mathrm{Ga}_{0.6} \mathrm{As} / \mathrm{GaAs}$ self-organized quantum dot lasers at room temperature," Appl. Phys. Lett., vol. 81, pp. 3055-3057, 2002.

[6] T. Akiyama, M. Ekawa, M. Sugawara, H. Kuwatsuka, H. Sudo, K. Kawaguchi, A. Kuramata, H. Ebe, and Y. Arakawa, "Recent advances in quantum-dot semiconductor optical amplifiers for telecommunications," presented at the 30th Eur. Conf. Optical Communication, 2004, Paper Mo3.4.1.

[7] N. Hatori, K. Otsubo, M. Ishida, T. Akiyama, Y. Nakata, H. Ebe, S. Okumura, T. Yamamoto, M. Sugawara, and Y. Arakawa, " $20{ }^{\circ} \mathrm{C}-70{ }^{\circ} \mathrm{C}$ temperature independent $10 \mathrm{~Gb} / \mathrm{s}$ operation of a directly modulated laser diode using p-doped quantum dots," presented at the 30th Eur. Conf. Optical Communication, 2004, Paper Th4.3.4.

[8] M. Kuntz, G. Fiol, M. Lummin, C. Schubert, A. R. Kovsh, A. Jacob, A. Umbach, and D. Bimberg, "10 Gbit/s data modulation using $1.3 \mu \mathrm{m}$ InGaAs quantum dot lasers," Electron. Lett., vol. 41, no. 5, pp. 244-245, Mar. 2005.

[9] P. Borri, W. Langbein, J. Mørk, and J. M. Hvam, "Heterodyne pumpprobe and four-wave mixing in semiconductor optical amplifiers using balanced lock-in detection," Opt. Comm., vol. 169, p. 317, 1999.

[10] M. V. Maximov, "Tuning quantum dot properties by activated phase separation of an InGa(Al)As alloy grown on InAs stressors," Phys. Rev. B, vol. 62, pp. 16671-16680, 2000

[11] O. Stier, M. Grundmann, and D. Bimberg, "Electronic and optical properties of strained quantum dots modeled by 8-band k-p theory," Phys. Rev. B, vol. 59, pp. 5688-5701, 1999.

[12] E. Gehrig and O. Hess, "Mesoscopic spatiotemporal theory for quantum-dot lasers," Phys. Rev. A, vol. 65, pp. 033804/1-033804/16, 2002 .
[13] J. Schilp, T. Kuhn, and G. Mahler, "Electron-phonon quantum kinetics in pulse-excited semiconductors: Memory and renormalization effects," Phys. Rev. B., vol. 50, pp. 5435-5447, 1994.

[14] T. Inoshita and H. Sakaki, "Electron relaxation in a quantum dot: significance of multiphonon processes," Phys. Rev. B, vol. 46, pp. 7260-7263, 1992.

[15] A. V. Uskov, J. McInnerney, F. Adler, H. Schweizer, and M. H. Pilkuhn, "Auger carrier capture kinetics in self-assembled quantum dot structures," Appl. Phys. Lett., vol. 72, pp. 58-60, 1998.

[16] J. L. Pan and P. L. Hagelstein, "Collisional processes involved in the population kinetics of semiconductor quantum-dot lasers," Phys. Rev. B, vol. 49, pp. 2554-2575, 1994.

[17] A. V. Uskov, K. Nishi, and R. Lang, "Collisional broadening and shift of spectral lines in quantum dot lasers," Appl. Phys. Lett., vol. 74, pp. 3081-3083, 1999.

[18] I. Magnusdottir, S. Bischoff, A. V. Uskov, and J. Mørk, "Geometry dependence of Auger carrier capture rates into cone-shaped self-assembled quantum dots," Phys. Rev. B, vol. 67, pp. 205326/1-205326/4, 2003.

[19] F. Borri, W. Langbein, J. M. Hvam, F. Heinrichsdorff, M.-H. Mao, and D. Bimberg, "Spectral hole-burning and carrier-heating dynamics in InGaAs quantum-dot amplifiers," IEEE J. Sel. Topics Quant. Electron., vol. 6, no. 3, pp. 544-551, May/Jun. 2000.

[20] R. Ferreira and G. Bastard, "Phonon-assisted capture and intradot Auger relaxation in quantum dots," Appl. Phys. Lett., vol. 74, p. 2818, 1999.

[21] T. Akiyama, H. Kuwatsuka, T. Simoyama, Y. Nakata, K. Mukai, M. Sugawara, O. Wada, and H. Ishikawa, "Ultrafast nonlinear processes in quantum-dot optical amplifiers," Opt. Quant. Elec., vol. 33, pp. 927-938, 2001.

[22] T. C. Newell, D. J. Bossert, A. Stintz, B. Fuchs, K. J. Malloy, and L. F. Lester, "Gain and linewidth enhancement factor in InAs quantum-dot laser diodes," IEEE Photon. Technol. Lett., vol. 11, no. 12, pp. 1527-1529, Dec. 1999.

[23] A. A. Ukhanov, A. Stintz, P. G. Eliseev, and K. J. Malloy, "Comparison of the carrier induced refractive index, gain, and linewidth enhancement factor in quantum dot and quantum well lasers," Appl. Phys. Lett., vol. 84 , pp. $1058-1060,2004$.

[24] P. M. Smowton, E. J. Pearce, H. C. Scheider, W. W. Chow, and M. Hopkinson, "Filamentation and linewidth enhancement factor in InGaAs quantum dot lasers," Appl. Phys. Lett., vol. 81, pp. 3251-3253, 2002.

[25] M. van der Poel, D. Birkedal, J. Hvam, and M. Lämmlin, "Alpha parameter in quantum-dot amplifier under optical and electrical carrier modulation," in Proc. Conf. Lasers and Electro-Optics (CLEO), vol. 1, May 2004, pp. 662-664.

[26] S. Schneider, P. Borri, W. Langbein, U. Woggon, R. L. Sellin, D. Ouyang, and D. Bimberg, "Linewidth enhancement factor in InGaAs quantum-dot amplifiers," IEEE J. Quantum Electron., vol. 40, no. 10, pp. 1423-1429, Oct. 2004.

Mike van der Poel received the M.Sc. degree in physics and the Ph.D. degree from the University of Copenhagen, Copenhagen, Denmark, in 1997 and 2001, respectively. His dissertation was on laser cooling and atomic collisions.

Since 2001, he has been an Assistant Professor at the Communication, Optics and Materials (COM) Research Center, Technical University of Denmark, Lyngby, Denmark. His research includes characterization of semiconductor components with emphasis on ultrafast spectroscopy of semiconductor optical amplifiers.

Edeltraud Gehrig received the diploma and Dr.rer.nat degrees in physics from the University of Kaiserslautern, Kaiserslautern, Germany, in 1995 and 1999, respectively.

From 2000 to 2003, she was a Senior Research Associate with the Theoretical Quantum Electronics Group, DLR Stuttgart, Stuttgart, Germany. Since April 2003, she has been a Senior Research Associate with the University of Surrey, Surrey, U.K. Her research includes biophotonic and biodynamic systems, molecular motors, ultrafast nonlinear dynamics, dynamics and control of high-power semiconductor lasers and amplifiers, luminescence, and quantum-dot lasers. 
Ortwin Hess received the Dipl.Phys. and Dr.rer.nat degrees in physics at the Technical University of Berlin, Berlin, Germany, in 1990 and 1993, respectively.

From 1990 to 1992 he was a Research Associate at Heriot-Watt University, Edinburgh, U.K., and the University of Tucson, Tuscon, AZ, and was a Postdoctoral Researcher at University of Marburg (1993-1994), Marburg, Germany. From 1994 to 2003, he was Head of the Theoretical Quantum Electronics Group, Institute of Technical Physics, DLR Stuttgart, Stuttgart, Germany. He has a Habilitation in Theoretical Physics at the University of Stuttgart, Stuttgart, Germany (1997), and became Adjunct Professor in 1998. Since 2001, he has been Honorary Professor at Tampere University of Technology, Tampere, Finland. He was a Visiting Professor at Stanford University, Stanford, CA (1997-1998), and the University of Munich, Munich, Germany (2000-2001). Since March 2003, he has held the Chair of Computational Quantum Electronics, Advanced Technology Institute. He is Head of the Theory and Advanced Computation Group, University of Surrey, Surrey, U.K. His research interests are focused on the theory and mathematical modeling of the ultrafast optics and complex laser dynamics (quantum-dot lasers, control of spatiotemporal laser dynamics, ultrafast effects in active semiconductor media), nanophotonic materials (quantum dots, photonic crystals, synthetic opals, liquid crystals, biomolecules), biomodeling, as well as nanothermodynamics and nanorheology. Together with his research group he strives to computationally explore the terahertz world and nanospace of novel advanced technologies in photonics as well as nano- and biomedical electronics.
Dan Birkedal received the M.Sc. and Ph.D. degrees in electrical engineering from the Technical University of Denmark (Electromagnetics Institute), Lyngby, Denmark, in 1987 and 1992, respectively.

In 1989, he was with the Department of Electrical Engineering and Material Science, University of Utah, Salt Lake City. He held a postdoctoral position (1992-1993) and was Assistant Research Professor (1994-1995) and Associate Research Professor (1995-1997 at the Microelectronics Center, Technical University of Denmark. In 1997-1999, he was a Visiting Scientist at Bell Laboratories, Lucent Technologies, Holmdel, NJ. In 1999-2004, he was an Associate Research Professor at Research Center Communication, Optics and Materials (COM), Technical University of Denmark. He is currently the Chief Technological Officer at Alight Technologies, Denmark.

Jørn M. Hvam received the M.Sc. degree in electrical engineering and the Ph.D. degree in physics from the Technical University of Denmark, Lyngby, Denmark, in 1969 and 1971, respectively.

From 1971 to 1993 , he held various positions at universities and research laboratories in Denmark, France, and the United States. Since 1995, he has been a Full Professor at the Technical University of Denmark where he currently heads the Nanophotonics at the Communication, Optics and Materials (COM) Research Center. His scientific research concerns the optical and optoelectronic properties of semiconductors. Over the last decade he has been mainly engaged in the fabrication and optical characterization of low-dimensional semiconductors, with special emphasis on ultrafast spectroscopy and dynamics of semiconductor nanostructures and devices. He heads national research programs on Semiconductor Components for Optical Signal Processing (SCOOP) and Nano Systems Engineering (NANOS). He has authored and coauthored more than 275 scientific papers appearing in international journals and books. He has given numerous invited talks and lectures at international conferences, meetings, and summer schools. 